\title{
Expériences répressives et (dé)radicalisation militante
}

La variation des effets de la répression sur les jeunes membres du Parti communiste français (1947-1962)

Repressive Experiences and (de)radicalization: the Consequences of Repression on Young Members of the French Communist Party (1947-1962)

\section{Vanessa Codaccioni}

\section{(2) OpenEdition}

\section{Journals}

\section{Édition électronique}

URL : http://journals.openedition.org/conflits/18629

DOI : $10.4000 /$ conflits. 18629

ISSN : $1777-5345$

Éditeur :

CECLS - Centre d'études sur les conflits - Liberté et sécurité, L'Harmattan

Édition imprimée

Date de publication : 15 juin 2013

Pagination : 29-52

ISBN : 978-2-343-01116-5

ISSN : 1157-996X

\section{Référence électronique}

Vanessa Codaccioni, «Expériences répressives et (dé)radicalisation militante », Cultures \& Conflits [En ligne], 89 | printemps 2013, mis en ligne le 15 juin 2014, consulté le 31 mars 2021. URL : http:// journals.openedition.org/conflits/18629; DOI : https://doi.org/10.4000/conflits.18629 


\section{Expériences répressives et (dé)radicalisation militante}

La variation des effets de la répression sur les jeunes membres du Parti communiste français (1947-1962)

\section{Vanessa CODACCIONI}

Vanessa Codaccioni est mâ̂tresse de conférences à l'Université Paris 8. Elle a notamment publié sur la mémoire communiste de la guerre d'Algérie (Terrains\&Travaux, 2010), sur le viol et l'affaire Djamila Boupacha en 1960-1962 (Nouvelles Questions Féministes, 2010) et, plus récemment, sur le rôle des avocats du Parti communiste français (PCF) dans le contexte répressif de la guerre froide (Le Mouvement social, 2012). Ses recherches actuelles portent sur la criminalité et la répression politiques depuis les années soixante-dix. Son dernier ouvrage, Punir les opposants. PCF et procès politiques 1947-1962, vient de paraitre aux éditions du CNRS.

$\mathrm{D}$

ans l'enquête réalisée pour ma thèse de doctorat sur les « procès politiques »communistes pendant le conflit indochinois et la guerre d'Algérie ${ }^{1}$, la répression est apparue comme un élément central du discours communiste, tous les membres du PCF interrogés - une cinquantaine - ayant souligné la véritable « hystérie » anticommuniste des agents de l'État et en particulier de la police, décrite comme brutale, agressive, et qui « tapait facilement 2 ». Si certains d'entre eux avaient été précisément choisis pour avoir été réprimés dans les années cinquante, constituant par là même une sorte de biais méthodologique, tous n'avaient pas été interrogés à cette fin. L'entretien avec l'ancien responsable CGT et sénateur Serge Boucheny par exemple, qui me demande de "bien marquer ça ${ }^{3}$ " (que les communistes étaient «très réprimés » pendant la guerre froide) avait pour objectif de revenir sur l'activisme anticolonial à l'intérieur des usines. Rien ne permettait de prévoir qu'il avait été arrêté à plusieurs reprises (sept fois dans cette période) dans le cadre de son

1. Codaccioni V., Punir les opposants. Une sociologie historique des " procès politiques " communistes en temps de crise : les interactions répressives entre le PCF et l'État (1947-1962), Thèse pour le doctorat de science politique, Université Paris 1, 2011, 699p.

2. Entretien avec Jacques Bonnet, Montpellier, le 2 janvier 2008.

3. Entretien avec Serge Boucheny, Paris, le 14 mars 2008. 
engagement politique. Les chiffres sont d'ailleurs parlants puisque hormis les responsables ou les militants déportés ou emprisonnés pendant la Seconde Guerre mondiale (cinq d'entre eux), plus de la moitié des enquêtés ont connu au moins un événement répressif entre 1947 et 1962. Subir la répression, tout au moins peut-on le supposer, était donc une expérience largement partagée par les membres du PCF dans les années cinquante. Néanmoins, plusieurs autres hypothèses complémentaires peuvent être faites pour expliquer cette insistance systématique des enquêtés sur la répression. Premièrement, les arrestations, les emprisonnements ou les jugements sont des moments émotionnellement forts dans la carrière militante des enquêtés et, même cinquante ans après les faits, un coup de matraque, une nuit au poste ou une convocation devant le juge d'instruction retiennent plus l'attention que la date précise d'une manifestation de rue. Bien entendu, cette présence de la répression dans le discours des enquêtés est aussi à relier aux mécanismes d'évocation des souvenirs en entretien et à « l'illusion biographique » propre aux récits sur soi, l'un et l'autre conduisant à choisir certains événements significatifs pour donner sens à une « histoire de vie ${ }^{4}$ ». Et si la répression est centrale dans les souvenirs des membres du PCF, c'est qu'elle permet de valoriser l'activisme communiste - forcément intense puisque réprimé - de souligner le fort anticommunisme qui sévissait pendant la guerre froide, mais aussi de justifier l'inaction communiste et les périodes de moindre engagement militant comme ce fut le cas, nous le verrons, lors de la guerre d'Algérie. Dans le même sens, et ceci est à rapporter aux observations de Fabien Jobard sur le caractère partial des récits de «violences policières » qui focalisent bien souvent l'attention sur un seul acteur de l'interaction - l'État ${ }^{5}$-, cette insistance sur la répression a tout autant pour but de délégitimer la politique du maintien de l'ordre mise en œuvre sous les IVe et ve Républiques, que d'euphémiser ou de justifier la violence communiste comme lors de la manifestation Ridgway de mai 1952.

C'est donc avec prudence qu'il faut mobiliser ces sources orales qui sont des reconstructions typiques des usages des souvenirs militants en entretien, notamment dans le cas du PCF où les discours de ses membres reflètent bien souvent, même des décennies après les faits, l'orthodoxie de parti intériorisée dans les années de lutte ${ }^{6}$. La correspondance entre leurs récits et le cadrage sur la répression «fasciste » et «bourgeoise » développé par le groupe dirigeant dans les années cinquante en est l'exemple le plus probant ${ }^{7}$, tout autant qu'il témoigne de la nostalgie d'un passé pourtant unanimement dénoncé : le

4. Bourdieu P., «L'illusion biographique », Actes de la recherche en sciences sociales, $\mathrm{n}^{\circ} 62-63$, 1986, pp. 69-72.

5. Jobard F., «Les violences policières », in Mucchielli L., Robert P. (eds.), Crime et sécurité. L'état des savoirs, Paris, Éditions La Découverte \& Syros, 2002, p. 206.

6. Pour la période de la guerre d'Algérie : V. Codaccioni., "Le futurisme historique c'est la catastrophe. La conflictualité de la mémoire de l'anticolonialisme communiste pendant la guerre d'Algérie ", Terrains E Travaux, n¹7, 2010, pp. 23-40.

7. Conclusion émise à partir du dépouillement des archives du PCF (Bureau politique, Secrétariat, Comité Central) et des organes de presse du PCF (dont L'Humanité) de 1947 à 1962. 
«passé stalinien 8 ». Mais, justement, si la répression est aussi présente dans le discours des enquêtés qui l'ont subie, c'est qu'elle fait partie intégrante de l'engagement communiste et que, conséquence subie ou recherchée de leur radicalité militante, elle est l'une des marques distinctives du PCF et associée à d'autres éléments constitutifs de sa singularité politique comme la nécessaire lutte contre l'État capitaliste ou la croyance en la future dictature du prolétariat. Elle a donc partie liée à l'histoire combattante, héroïque et glorieuse de ses membres, aux schèmes et aux pratiques qui régissent l'activité militante mais aussi au fonctionnement même de l'institution communiste qui se transforme, évolue et se modifie au gré de ce que j'ai appelé les «interactions répressives 9 » avec les agents de l'État. Or, si la répression est si stratégique dans le dispositif de mobilisation communiste, suscitant l'enthousiasme et le dynamisme militants comme lors des années cinquante, qu'en est-il dans les périodes de faibles affrontements avec les institutions répressives ? Comment l'organisation communiste et ses membres s'adaptent-ils aux variations des relations conflictuelles avec l'État, et en particulier aux périodes de moindre radicalité militante comme cela est le cas pendant la guerre d'Algérie ?

Suivre les militants communistes dans leurs confrontations avec les agents répressifs durant la guerre froide et le conflit algérien permet dès lors d'observer deux configurations successives de l'institution communiste, l'une marquée par la radicalité politique, l'autre par le légalisme, et de dégager l'évolution des rapports entre le PCF et l'État. Plus précisément, ce type d'analyse donne la possibilité de restituer le processus de « déradicalisation 10 » qui touche l'organisation partisane dès la fin de la guerre d'Indochine et d'éclairer la manière dont l'abandon de la stratégie d'affrontement direct avec l'État ouvre un espace à gauche, suscite le désengagement de certains de ses membres et inaugure un processus d'(auto)-exclusion des réseaux de l'avant-garde militante que viendront parfaire les événements de mai-juin 1968. Ainsi, dans cet article, centré sur les effets de la répression sur les militants communistes, leur organisation et sur les liens partisans, c'est plus généralement la question des coûts et des rétributions engendrés par les interactions répressives avec l’État qui sera interrogée plus avant.

À travers l'analyse des trajectoires de quelques jeunes membres du PCF, ceux qui sont réprimés pour la première fois dans les années cinquante, on verra dans un premier temps la manière dont la répression favorise la radicalisation militante, attache les jeunes à l'organisation et participe de la tenue d'un rôle, celui du « militant révolutionnaire » des périodes de repli, marquées entre

8. Pudal P., Un monde défait. Les communistes français de 1956 à nos jours, Paris, Éditions du Croquant, 2009, p. 8.

9. Sur cette notion, qui permet d'insister sur la dimension relationnelle de la répression et de la saisir comme le résultat des interactions entre les opposants et les agents de l'État, voir Codaccioni V., Punir les opposants..., p. 44-45.

10. Sur les processus de radicalisation/déralication politique et militante, voir Collovald A., Gaiti B., (eds.), La démocratie aux extrêmes. Sur la radicalisation politique, Paris, La Dispute, 2006. 
autres par le durcissement de la discipline partisane, la radicalisation des pratiques et des discours, et les affrontements violents avec les forces de l'ordre. Puis, dans un second temps, à travers le cas du conflit algérien, on s'intéressera à la manière dont l'abandon de la radicalité militante et la dévalorisation de l'expérience vécue de la répression ont des effets démobilisateurs sur les franges les plus radicalisées du parti, qui transgressent dès lors ses normes partisanes ou se désengagent pour lutter plus activement contre la guerre d'Algérie.

\section{Guerre froide et conflit indochinois : les jeunes au cœur du processus de radicalisation politique}

Dès le début de la guerre froide, le Parti communiste entre dans un processus continu de radicalisation militante induit par une multiplicité d'événements interdépendants (la grève des mineurs d'avril 1947, l'exclusion des ministres communistes du gouvernement le mois suivant, etc.) qui l'isolent du champ politique et font disparaître toute possibilité de réintégrer le pouvoir. Cette radicalisation, qui tient avant tout au contexte international de guerre froide et à sa retraduction dans les divers champs politiques occidentaux, doit aussi pour beaucoup à la modification progressive du degré de radicalité des membres du PCF qui, tout au long de cette période, mettent en œuvre des modalités non conventionnelles de lutte comme l'antimilitarisme de masse ou clandestin. La répression étatique, facilitée par un anticommunisme d’État sous-tendu par la théorie du «complot » et légitimée par l'activisme communiste, découle dès lors tout autant qu'elle entretient ce processus jusqu'à la fin de la guerre d'Indochine. Dans cette séquence militante où la répression est voulue, anticipée et suscitée par le groupe dirigeant à des fins politiques, elle constitue dès lors l'un des aspects essentiels de l'engagement communiste, surtout pour les nouveaux adhérents qui deviennent les cibles prioritaires des agents de l'État par le suractivisme déployé. Si leurs expériences répressives constituent de puissants vecteurs de socialisation au militantisme communiste, elles n'en ont pas moins d'autres effets, tant sur leurs pratiques d'engagement, qui se radicalisent au fur et à mesure des interactions avec les agents répressifs, que sur leurs trajectoires militantes puisqu'elle favorise l'intégration et l'attachement durables à l'organisation.

\section{Un suractivisme militant encouragé par la direction}

La relation dynamique entre le militantisme « jeune » et la répression, tout comme les effets de celle-ci sur la jeune génération qui adhère au PCF à partir de la Libération, peuvent être analysés à partir des trajectoires d'Henri Malberg et de Maurice Verdier.

Henri Malberg est né en janvier 1930 dans le 18e arrondissement dans une famille de juifs polonais venue en France dans les années vingt. Son père, 
ouvrier d'usine puis mineur pendant un temps, devient artisan, sa mère ne travaille pas, et s'ils ne sont pas communistes, ils lisent tous deux des journaux juifs socialisants. Henri Malberg a 9 ans quand la guerre éclate. Scolarisé à l'école de Belleville, il est alors obligé de se cacher et de fuir sous l'Occupation. La référence à la Résistance est dès lors centrale dans son discours, lui qui adhère « tout naturellement » aux Jeunesse communistes en 1945 :

«Il y a chez des jeunes comme moi l'admiration des communistes de la Résistance, dit-il. Moi j'avais 13 ans, eux en avaient 18. Et puis ma génération avait tenu le fusil [...]. Quand moi à 15, 16 ans je deviens communiste, il y a les héros de la Résistance. Les cercles de la jeunesse communiste dans le XIX et le XXe portent des noms de jeunes qui avaient 5 ans de plus que moi et qui avaient été tués ${ }^{11}$ ».

Ainsi, si l'adhésion d'Henri Malberg s'inscrit dans un flot d'adhésion dans lequel dominent les juifs et les étrangers réfugiés politiques ${ }^{12}$, elle illustre surtout le type d'adhésion communiste au sortir de la guerre, celle de jeunes que peu de choses prédisposaient à l'engagement communiste ou tout au moins de jeunes qui ne sont pas des « héritiers 13 » et qui adhèrent dans l'euphorie de la Libération à un Parti fort des « 75000 fusillés » sous l'Occupation. Mais ces nouveaux adhérents, qui n’ont bien souvent comme seule référence que la Résistance et ses grands hommes (Staline, Churchill ou de Gaulle), sont aussi pour la plupart sans formation politique et idéologique. Ils nécessitent dès lors un «énorme travail 14 » pour les cadres du PCF puisqu'ils vont devoir être formés, encadrés et socialisés aux discours et aux pratiques communistes, notamment au sein de ses organisations de jeunesse comme l'Union de la Jeunesse Républicaine de France (UJRF), créée en 1945, et à laquelle adhère Henri Malberg en 1947. Après l'éviction des ministres communistes du gouvernement en mai de cette année-là, il entame alors une nouvelle phase de son engagement, celle du militantisme de "guerre froide », qui donne lieu à de multiples arrestations et inculpations comme celle dont il écope en décembre 1950 pour « coups et blessures » et « rébellion » :

«Il faut comprendre que pour des gens de mon âge, la Libération de la France, le 8 mai 45, la marche dans Paris du peuple entier... il y avait comme un élan de bonheur. C'était un élan, la vie semblait nous appartenir. Et quand on était communiste, on avait le sentiment d'avoir été dans un truc qui avait été glorieux, qui était plein d'avenir. Et donc la guerre froide tombe comme un manteau de glace. Je me rappelle la première manif à Wagram où on se fait

11. Entretien avec Henri Malberg, Paris, le 17 janvier 2008.

12. Lavau G., À quoi sert le Parti communiste français, Paris, Fayard, 1981, p. 100.

13. Kriegel A., Les communistes français, Paris, Editions du Seuil, 1968, p. 93.

14. Selon les mots de Maurice Thorez à Staline le 18 novembre 1947 (Communisme, n 45-46, 1996, p. 47). 
matraquer par la police parce qu'on essayait d'empêcher un meeting, le premier meeting d'extrême droite. Eh bien, on criait “la police avec nous” et ils nous tapaient dessus [...]. Et là-dessus tombe la première guerre du Vietnam. Pour les gens comme moi, c'est une très très grande lutte qui nous occupe énormément. On vend l'Avant-Garde, on manifeste sur les marchés, on se fait... par exemple là dans le XIX où j'étais à l'époque, Place des Fêtes, tous les dimanches on était amenés par la police et on passait l'après-midi à 20, 30, 40 au commissariat. Tous les dimanches, les flics nous embarquaient, mais au point que on savait que vers 11 heures, midi, on ferait une petite manif, on déploierait une banderole, les flics rappliqueraient et ils nous embarqueraient. [...] Alors moi, du point de vue personnel là, je suis un des premiers jeunes qui va en prison. Parce qu'il y avait un peu l'idée "la guerre c'est pas seulement s'y opposer c'est faire des actes” [...]. Tiens, j’ai retrouvé ça l'autre jour [un article de presse 15], vous verrez. Et donc on s'est fait choper. On s'est couché devant les cars Place des Fêtes et ils nous ont embarqués pour "coups et blessures à agent". On a chopé un mois de taule avec sursis.

- Pourquoi vous vous êtes couchés devant les cars?

- Parce que les flics nous embarquaient tous les dimanches. Alors donc on a dit un jour... après je vous parlerais de la manif Ridgway... en nous montait la colère, la haine, on était sans cesse matraqués, embarqués et on avait dit :" Ce dimanche-là on empêche les cars de partir". Il y avait un gros car bourré de jeunes, comme toujours on criait : "La paix ! La paix !” Et alors on s'était mis tous les deux avec Samy devant les roues. Les flics nous ont tirés, nous ont foutu une rouste, et ensuite, pour nous faire peur, ils nous ont emmenés au dépôt, la fameuse souricière, qui est toujours aussi crade, pour rébellion ».

L'entretien avec Henri Malberg est tout à fait exemplaire pour illustrer le militantisme communiste des années de guerre froide, un activisme quotidien ponctué d'affrontements plus ou moins violents avec les forces de l'ordre et sous-tendu par la volonté de «faire des actes ». Il montre aussi comment, à travers la lutte contre la guerre d'Indochine, se forme toute une génération de jeunes communistes et en particulier éclaire la manière dont ils se socialisent aux pratiques d'un engagement vécu par eux (et construit par la direction à cette date) comme "révolutionnaire ». En effet, à cette période (1949-1950), tandis que le Kominform appelle les différents PC à être plus « offensifs 16 » et que les altercations entre communistes et agents répressifs se multiplient, se diffuse au sein du PCF une stratégie discursive axée sur la dénonciation de la

15. Il s'agit d'un article de Libération signé par Madeleine Riffaud.

16. Sommier I., Brugié J, Officier et communiste dans les guerres coloniales, Paris, Flammarion, 2005, p. 112. 
« justice bourgeoise » et de la «fascisation » de la police qu'incarnent les «persécuteurs » comme Jules Moch 17, Jean Baylot 18, ou les CRS avec « leur matraque, leurs mitraillettes et leur fusil 19 ». Il s'agit au sein du Parti de "développer la haine de classe vis-à-vis des policiers 20 » et de montrer que « la lutte contre la police est l'ABC du militant révolutionnaire », une recommandation de Lénine qui se propage dans le Parti via ses organes de presse ${ }^{21}$. Plus précisément en ce qui concerne la lutte contre la guerre d'Indochine, les dirigeants en appellent dès 1949 à " l'esprit d'initiative, de dévouement et de sacrifice 22 » des communistes, c'est-à-dire encouragent les pratiques militantes « ̀̀ risque » dont ils savent qu'elles vont être plus ou moins durement réprimées, comme les actions violentes et la lutte contre le transport et le départ du matériel de guerre vers le Vietnam. Des militantes et des militants se couchent dès lors sur les rails de trains chargés de matériel de guerre ou empêchent leur départ par le biais de manifestations plus ou moins violentes largement réprimées et valorisées dans la presse communiste. Ils deviennent dès lors des «victimes de la répression ", selon le cadrage héroïque déployé par le groupe dirigeant dès 1949 23, c'est-à-dire sont hérö̈sés pour mobiliser les militants sur des causes ponctuelles puissamment mobilisatrices (la libération ou l'acquittement des accusés) et pour servir de référence et de modèle d'identification aux plus jeunes d'entre eux. Leur assimilation à ceux « morts pour la France » ou tout au moins aux résistants communistes qui ont pris les armes contre l'occupant renforce ce processus d'héroïsation. On comprend ainsi comment l'expérience vécue de la répression est un événement qui permet de conforter le cadrage sur la répression illégitime de l'État « capitaliste » et «bourgeois » et d'intérioriser toute une vision de l'histoire interprétée en termes de répression, d'anticommunisme et «d'ennemis de classe ». Être réprimé devient ainsi un devoir militant, source de fierté et de satisfaction puisque correspondant aux nécessités de la « lutte» et aux directives de la direction. Elle représente alors une « rétribution militante » en soi car preuve d'un activisme " révolutionnaire » et d'une conformité au rôle prescrit par l'organisation. Henri Malberg fait d'ailleurs partie des militants qui restent au PCF tandis que ce dernier subit une forte décrue des effectifs ${ }^{24}$, c'est-à-dire qu'il est représentatif de ceux qui correspondent aux attentes du Parti, qui retirent des gratifications de leur engagement, et donc de ceux qui ont partiellement intégré l'orthodoxie et la discipline communistes ou sont prêts à s'y conformer.

17. Jules Moch, socialiste, ministre de l'Intérieur de 1947 à 1950, participe notamment à radicaliser la répression de la grève des mineurs de 1947.

18. Anticommuniste virulent, le socialiste Jean Baylot devient Préfet de police de Paris le 17 avril 1951.

19. L'Humanité, 11 janvier 1950.

20. Les Cabiers du communisme, $\mathrm{n}^{\circ} 5$, mai 1951.

21. Idem.

22. Arch. PCF, Séance du Bureau politique du 23 septembre 1949.

23. Arch. PCF, Séance du Secrétariat du 25 octobre 1949.

24. Le PCF subit une perte de 40000 adhérents en 1947 (4,9\% de ses effectifs), puis de 450000 entre 1948 et 1952 (57,4 \%) (Buton P., "Les effectifs du Parti communiste français », Communisme, $1985, \mathrm{n}^{\circ} 7$, p. 16). 
À ceux qui « restent ", malgré le contexte de guerre froide, la radicalisation des discours, le renforcement de la discipline de Parti et du contrôle idéologique ou encore la généralisation de la répression, s'ajoutent ceux qui s'engagent au PCF pendant la guerre froide. Ces derniers sont alors soit des fils ou des filles de communistes prédisposés à l'engagement au sein du Parti, soit des jeunes déjà politisés «à gauche » et qui voient dans le PCF un parti « d'avantgarde » ou le seul capable de défendre certaines causes comme par exemple l'anticolonialisme. C'est ce que Georges Lavau appelle les « adhésions de combat » qui sont le fait d'hommes ou de femmes « résolus à lutter, prêts à se faire mettre en quarantaine, à se faire insulter, à ne pas fléchir, fiers d'être "staliniens" et "cocos" ou encore heureux de haïr à plein poumons les Moch, les Mollet, les Schuman, les Pleven 25 ». Ce type d'adhésion peut être illustré par le cas de l'ancien dirigeant fédéral de l'Hérault, Maurice Verdier, que j'ai rencontré chez lui à Béziers.

Maurice Verdier est né à Montpellier en 1924. Dès ses 4 ans, il séjourne au Sénégal où son père, membre de la Section française de l'internationale ouvrière (SFIO) du Sénégal, est fonctionnaire colonial. Ce «nègre blanc » comme il se nomme lui-même, est alors confronté à la réalité coloniale:

\begin{abstract}
«J'ai vu le colonialisme dans toute son horreur. Mon père, qui était socialiste et tout, n'aurait jamais reçu un noir à sa table [...]. Je voyais le spectacle des types qui tapaient sur les prisonniers à coup de cravache, et les prisonniers enchaînés qui travaillaient dans la concession, ils avaient une chaîne autour du cou et une au pied pour qu'ils ne s'évadent pas. Alors là, ça m’a un peu éveillé quand même».
\end{abstract}

Après avoir obtenu à Dakar son Brevet d'aptitude coloniale (équivalent du baccalauréat), Maurice Verdier revient en 1945 à Montpellier pour y étudier le droit avec une bourse de l'Afrique-Occidentale française (AOF). Lors d'un voyage d'été au Sénégal en 1947, il prend sa carte au Rassemblement Démocratique Africain ${ }^{26}$, premier acte d'un engagement anticolonialiste, et, rentré en France, adhère au PCF au moment de la grève des mineurs, l'un des moments répressifs les plus décisifs de la période puisqu'il aboutit à l'exclusion des ministres du gouvernement en mai 1947 et à l'éviction durable du PCF du champ politique central. Les adhésions de la guerre froide, dans le cas de jeunes qui ne sont pas des « héritiers » mais dont les trajectoires biographiques et les fréquentations militantes favorisent l'engagement communiste, diffèrent donc sensiblement de celles de l'après-guerre puisqu'elles reposent sur une meilleure connaissance de l'idéologie partisane et ne visent pas à intégrer un parti au pouvoir mais une organisation de plus en plus stigmatisée et

25. Lavau G, À quoi sert le Parti communiste français, op. cit., pp. 100-101.

26. Le RDA est un mouvement politique pour toute l'Afrique francophone créé en 1946 par Félix Houphouët-Boigny. 
réprimée. En 1950, Maurice Verdier devient journaliste à la rédaction du journal communiste montpelliérain, La Voix de la Patrie, avant d'être promu, avant la fin de la guerre d'Indochine, chef de l'agence locale du quotidien communiste La Marseillaise. À propos de la guerre d'Indochine, Maurice Verdier nous décrit son engagement comme une période où il «s'amusait bien » malgré son inculpation «d'atteinte au moral de l'armée » pour distribution de tracts contre le conflit colonial :

"J’ai fait le con ${ }^{27}$. Au début... mais j'ai été vite repéré par les dirigeants de l'époque, d'abord parce que j'étais de tous les manvais coups. À cette époque, on distribuait des tracts aux soldats contre la guerre d'Indochine, j'ai été inculpé, arrêté par la police et tout. On distribuait des tracts, on faisait des manifestations à Montpellier contre la guerre d'Indochine. On a même... Je vous raconte une anecdote. Il y avait une cérémonie où on donnait la légion d'honneur au ministre de la guerre Coste-Floret. Il est connu. Et nous, avec les copains, on a fait monter sur le thêâtre de Montpellier une énorme banderole "Paix en Indochine ", avec un système qui date de la Résistance, un système de tapette à rat qui a un réveil. [...] Et alors [il rit] au milieu de la cérémonie, tout le monde a tourné la tête, et l'énorme banderole s'est déployée. Les flics étaient fous, tout le monde... C'étaient des copains un peu acrobates qui avaient mis ça sur le haut du thêâtre. C'étaient nos jeux. Et après, comme distraction, on allait coller les affiches contre la guerre d'Indochine sur les cars de police. Et alors, le maton, il était dans le bureau du secrétaire fédéral qui était Balmigères pour se plaindre des jeunesses communistes, et une heure après on était convoqués dans le bureau du secrétaire fédéral. Il disait: "Qu'est-ce que vous avez fait encore comme connerie ?" [il rit]. C'était... On était révolutionnaires pour de bon. Maintenant il n'y en a plus.

- Et là vous avez été arrêté pour les tracts?

- J'ai été inculpé [...], les flics me cherchaient partout pour me faire signer le non-lieu. Mais comme on était anti-justice, $j$ 'ai toujours refusé de signer quelque chose. C'était pas grave. C'était une époque où on s'amusait bien $28 \%$.

Les deux cas que nous venons de décrire sont représentatifs du militantisme anticolonial communiste et plus généralement de la « lutte pour la paix » chez de jeunes militants récemment membres du PCF, prêts à « faire des actes » (Malberg) ou, sur un ton plus ludique, à « faire le con » (Verdier). Si l'aspect idéologique du militantisme communiste et l'attachement à une cause favorisent l'engagement « de guerre froide », leurs exemples donnent aussi à

27. Nous soulignons.

28. Entretien avec Maurice Verdier, Béziers, le 20 décembre 2007. 
voir le « caractère plaisant 29 » des activités anticoloniales pour les plus jeunes membres du PCF. Cette véritable atmosphère de jeu permet alors, parallèlement à la répression subie, de les socialiser à l'agit-prop et aux savoir-faire qui lui sont associés (tractage, collages d'affiches ou déploiements de banderoles etc.) voire de consolider par leur caractère collectif - avec « les copains » - la cohésion partisane. Dans ce cas, l'expérience vécue de la répression, quotidienne, ordinaire et banale mais aussi sans véritable coût 30 (les sanctions sont rares et se caractérisent souvent par de faibles amendes ${ }^{31}$ ) - ce n'est « pas grave " se souvient Maurice Verdier - participe ainsi de l'entretien de l'enthousiasme et du dynamisme militants. Néanmoins, et ce point est parfaitement visible à la fois dans l'entretien réalisé avec Henri Malberg - qui me remet un article rédigé par Madeleine Riffaud sur son arrestation - ou dans le témoignage de Maurice Verdier qui se souvient avoir été « repéré » par les dirigeants pour avoir été «de tous les mauvais coups », l'expérience de la répression peut aussi avoir des incidences plus ou moins fortes sur la trajectoire des militants réprimés. En effet, si pour les cadres et les dirigeants communistes, déjà connus et reconnus au sein du PCF, l'expérience de la répression ne fait qu'accroître leur capital de notoriété et renforcer leur domination charismatique au sein du Parti ${ }^{32}$, elle est pour un jeune militant l'une des rares occasions de voir son nom cité en exemple dans les colonnes de L'Humanité, de susciter l'admiration ou la sympathie des "camarades ", et parfois même de " monter» dans la hiérarchie 33. Si le cas du " héros » Henri Martin ${ }^{34}$, condamné à cinq ans d'emprisonnement pour distribution de tracts antimilitaristes est le plus connu - lui qui devient permanent l'année suivant sa libération (1954) et Secrétaire général des jeunesses communistes en 1956 - c’est l'ensemble de cette nouvelle génération qui constitue un vivier de potentiels cadres et dirigeants qui font défaut à l'organisation. Cependant, ces usages juvéniles de la violence, décrits à partir des cas d'Henri Malberg ou de Maurice Verdier, typiques des groupes d'extrême gauche 35 et qui attachent les jeunes à l'institution, renforcent aussi tout autant qu'ils entretiennent un « sentiment d'adversité » face aux « autres » (les anticommunistes, les agents répressifs). Ils sont ainsi un facteur d'intériorisation d'une discipline rigoureuse et les vec-

29. Sur le caractère ludique de la socialisation politique au sein des organisations de jeunesse, voir Bargel L., Jeunes socialistes, jeunes UMP : lieux et processus de socialisation politique, Paris, Dalloz, 2009.

30. En analysant les expériences répressives du PCF dans cette période, on observe en effet une correspondance entre degré de répression et degré d'intégration dans le Parti. Les jeunes par exemple, sans réelle responsabilité partisane et pratiquant un "militantisme de propagande » sont ainsi moins réprimés (en termes de gravité des « crimes » attribués et des sanctions prononcées) que les cadres ou les dirigeants du PCF. Sur ce point, voir Codaccioni V., Punir les opposants..., op. cit.

31. Conclusions émises à partir du dépouillement des archives judiciaires sous la côte BB18.

32. Idem., p. 334-336.

33. Idem., p. 370.

34. Ruscio A (ed)., L'affaire Henri Martin et la lutte contre la guerre d'Indochine, Pantin, Le temps des Cerises éditeurs, 2005.

35. Yon K., « Modes de sociabilité et entretien de l'babitus militant. Militer en bande à l'AJSOCI », Politix, 2005, n 70, pp. 148-152. 
teurs d'une radicalisation politique progressive, qui se construit au gré de la multiplication des interactions répressives avec les agents de l'État.

\section{Expérience répressive et radicalisation militante : le climax répressif de la manifestation Ridgway de mai 1952}

La manifestation Ridgway du 28 mai 1952, organisée dans le contexte du conflit coréen et des débats sur la guerre bactériologique ${ }^{36}$ contre la venue à Paris du général américain Matthew Bunker Ridgway, est tout à fait révélatrice de la violence exponentielle des manifestations communistes. Henri Malberg qui, en revenant du service militaire, devient en 1952 permanent de l'Union de la jeunesse républicaine de France (UJRF), raconte :

" “On manifeste, et on montre qu'il y en a marre de se faire casser la gueule”. Et c'était très très bien pris. On se disait : “Ce coup-là, on ne nous aura pas" 37 ».

La manifestation Ridgway est alors organisée à des fins d'affrontements voulus avec les forces de l'ordre : les militants fabriquent des armes avec des punaises, des boulons ou des clous, et ils s'arment eux-mêmes avec des bâtons, des triques, des armes à feu ou des haches. Parmi eux se trouve Serge Magnien, qui représente la figure type de « l'héritier ». Né en 1929 dans une famille communiste, sa mère est, jusqu'à la Seconde Guerre mondiale, directrice de l'école russe de Paris, et son père, Marius Magnien, est ancien responsable de la politique extérieure à L'Humanité. Après avoir adhéré aux Jeunesses communistes à la Libération, il prend sa carte du Parti en 1949 et milite activement dans la section du $6^{\mathrm{e}}$ arrondissement dont il devient rapidement le secrétaire. Comme nombre de jeunes pendant la guerre froide, il est appréhendé de nombreuses fois par les forces de l'ordre entre 1949 et 1953 : en avril 1949, alors qu'il vend L'Avant-garde, deux mois plus tard lors d'une manifestation communiste contre la projection du film Le rideau de fer, ou encore en juin 1953 lors d'un meeting du PCF à la Mutualité ${ }^{38}$. Au moment de la manifestation Ridgway, il est étudiant aux Beaux-arts et rédacteur en chef de Clarté, le journal des étudiants communistes :

«-Vous vous rappelez de la manifestation Ridgway?

- Ridgway oui [il rit beaucoup]. Petite pancarte "Non à Ridgway, Ridgway home", et le bâton dans le pantalon [...]. Je sors ma petite boîte de punaises, deux punaises sur le bâton, ma petite pancarte et vas-y, vas-y cocotte. Et on s'est retrouvés place de l'Odéon, il y avait la police qui était là mais ils n'étaient pas très nombreux. Et le

36. Matonti M., « La colombe et les mouches. Frédéric Joliot-Curie et le pacifisme des savants », Politix, 2002, n 58, pp. 109-140.

37. Entretien avec Henri Malberg déjà cité.

38. Selon un rapport militaire : Arch. SHD, 10T515. Note de renseignements du 5 mars 1958. 
malheur veut que le commissaire principal du $6^{\mathrm{e}}$ arrondissement, le commissaire M. était là à côté d'une voiture de police. Il y avait des copains qui le connaissaient. Et puis la masse des manifestants a coincé M. dans un petit bistrot qui existe encore. Il est tombé assis sur une chaise à la terrasse, une chaise en osier et les gars ont commencé à le battre, mais vraiment un lynchage. Et moi j'étais à côté, toujours avec ma petite pancarte et quand j'ai vu le sang venir à la figure, là on s'est précipités à deux ou trois, je n'étais pas tout seul, pour arrêter le massacre. Le type, on l'a dégagé, on l'a laissé dans sa chaise et les gars se sont rabattus sur la voiture de police, ils ont essayé de foutre le feu. Et à l'intérieur il y avait le chauffeur, il se défendait avec la manivelle. Après on a traversé la Seine [...] et on s'est engagés dans la rue de la Banque. Et là, il y avait deux camionnettes de police. On a essayé de renverser la voiture de police, il faisait déjà tard [...] et les gars qui étaient dans le commissariat ont commencé à tirer à la mitraillette sur le mur d'en face. J'ai entendu des coups de feu, il y a un gars qui a été blessé, on l'a traîné jusque devant la Bourse. On a arrêté une voiture, on a mis dedans le gars qui pissait le sang et on a dit: "Emmenez-le à l'hôpital". [...] Voilà, c'est mon souvenir de Ridgway 39 ».

L'extrait d'entretien avec Serge Magnien, s'il permet de saisir un peu mieux ce que fut la manifestation Ridgway, une manifestation dans laquelle dominent les hommes, les jeunes adultes et les ouvriers, illustre la violence des mobilisations du 28 mai, une violence qui, expression d'un «éthos de virilité exacerbée 40 » peut être l'un des principaux facteurs de participation à des rassemblements de ce type. C'est d'ailleurs ce que souligne Danielle Tartakowsky lorsqu'elle écrit, à propos de cette manifestation, que la violence contre les forces de l'ordre « constitue en elle-même sa propre fin » ou que « la rue redevient une fin en soi 41 ». Le recours à la violence et ses effets sur les militants, à savoir le possible entretien d'une "représentation guerrière 42 » qu'ils ont d'eux-mêmes ou tout simplement la satisfaction de « tenir la rue », expliquent aussi la vivacité des souvenirs qui lui sont liés chez ceux qui y ont participé. Ces souvenirs sont d'ailleurs beaucoup plus présents que les directions successives ne le souhaiteraient ${ }^{43}$ puisqu'ils illustrent une "ligne ultragauche » vite abandonnée et qu'ils soulignent tous la violence des participants et donc la part de responsabilité communiste dans la répression subie. Car les coûts de la répression sont très lourds pour le mouvement communiste. On dénombre deux morts, plusieurs centaines de militants arrêtés - 718 à Paris

39. Entretien avec Serge Magnien, Paris, le 1er février 2008.

40. Mauger G., «Les mondes des jeunes », Sociétés contemporaines, 1995, n 1, p. 13.

41. Tartakowsky D., Les manifestations de rue en France, 1918-1968, Paris, Publications de la Sorbonne, 1997, p. 569.

42. Mauger G., Fossé-Polliak C, «La politique des bandes », Politix, 1991, n 14, pp. 32-33.

43. Pigenet M, Au cour de l'activisme communiste de la guerre froide..., op. cit., p. 8. 
dont le numéro 2 du PCF, Jacques Duclos, inculpé de « complot », 292 dans le reste de la France et de nombreuses hospitalisations - des cadres et des dirigeants décident de "prendre la clandestinité 44 » pour se soustraire à la Justice, et des «secrets de Parti », comme les listes d'adhérents ou les biographies de ses cadres, sont découverts lors de perquisitions effectuées par les forces de l'ordre. Ce coût de la répression, le plus élevé depuis l'Occupation et vécu comme tel par les dirigeants du PCF et ses membres, explique ainsi en partie la disparition de la «violence communiste » à partir de cette date et le recul de l'activisme anticolonial communiste pendant la dernière année du conflit indochinois et, plus encore, pendant la guerre d'Algérie.

Ce que nous venons de décrire à propos de la guerre froide, à savoir la manière dont la répression découle de la radicalisation militante tout autant qu'elle l'entretient, et dont elle participe du renforcement de l'engagement partisan, ne vaut que pour les périodes où le $\mathrm{PCF}$, sévèrement réprimé et exclu du gouvernement, souhaite utiliser la répression à des fins politiques. À chaque cycle répressif, comme à la fin des années vingt, des années trente et sous l'Occupation, elle représente ainsi une composante essentielle du militantisme PCF et une étape majeure dans la carrière de ses membres. Mais dans les séquences où la participation au pouvoir (re)devient un horizon possible, comme lors de la guerre d'Algérie, ce n'est plus le fait d'être réprimé qui est gratifié et gratifiant mais la capacité à respecter les consignes légalistes du groupe dirigeant.

\section{La guerre d'Algérie : la résistance des jeunes à l'abandon de la radicalité militante}

La guerre d'Algérie ouvre en effet une tout autre configuration socio-politique et judiciaire dans laquelle le nombre des interactions répressives entre les membres du PCF et les agents de l'État diminue considérablement. Cette situation, qui s'explique avant tout par le légalisme communiste et, incidemment, par la dévaluation de la dangerosité politique du PCF par rapport aux nationalistes algériens et à d'autres formations anticoloniales aux modalités d'actions plus radicales, modifie les pratiques d'engagement des membres du PCF et le rapport qu'ils entretiennent aux expériences vécues de la répression, plus rares et délégitimées par les instances dirigeantes du Parti. Dans ce contexte d'affaiblissement de la radicalité militante et de la conflictualité avec les agents répressifs, les liens partisans entre le groupe dirigeant et certains de ses membres qui veulent radicaliser la lutte contre le conflit algérien se fragilisent et se distendent. Les jeunes en particulier, nouveaux adhérents ou militants depuis la guerre froide directement confrontés à la guerre par les mesures

44. L'expression est empruntée à Gaston Viens qui, secrétaire fédéral des Bouches-du-Rhône au moment des faits, ne se présente pas devant le juge d'instruction (Entretien avec Gaston Viens, Orly, 18 janvier 2008). 
de rappel des soldats en deux vagues en 1955 et 1956, tendent à transgresser les normes militantes communistes en désertant, en refusant de porter les armes ou plus généralement en radicalisant leur modalité d'opposition à la guerre d'Algérie. L'analyse de leur rapport à la répression, eux qui sont devenus des dissidents, des « hors-ligne » ou des «ex » récents démissionnaires et exclus, permet dès lors de souligner un autre aspect du lien entre militantisme et répression, celui des rapports de causalité entre les expériences répressives, la désobéissance partisane et le désengagement militant.

\section{L'abandon de la violence manifestante et la dévalorisation des expériences répressives}

Dès novembre 1954, date du déclenchement de la guerre d'Algérie, le groupe dirigeant du PCF opte, dans une volonté d'intégrer le champ politique et par crainte d'une répression généralisée notamment, pour une ligne légaliste qui perdure officiellement jusqu'à la fin de la guerre ${ }^{45}$. Dans ce contexte d'abandon de la radicalité militante, toute action politique potentiellement réprimée par les agents de l'État est interdite par les instances dirigeantes du parti. Il en va ainsi de toutes les pratiques «à risque » mobilisées pendant la guerre froide comme l'antimilitarisme de masse mais aussi les violences exercées contre les forces de l'ordre. Hormis les manifestations contre les rappels de soldats en 1955 et 1956 qui aboutissent à l'arrestation de plus de soixantedix membres du parti dont certains jugés par le tribunal militaire, les mobilisations communistes de la guerre d'Algérie se caractérisent dès lors par leur caractère pacifique et non-violent. Plus spécifiquement, ce sont des manifestations rapides et mobiles dont l'objectif premier est d'éviter l'arrestation des militants, comme me le raconte Alain Roux, responsable de l'Union des Étudiants communistes (UEC) :

"On faisait la queue dans tous les cinémas du quartier, et à un moment donné on bloquait le Boul' Mich au milieu, on faisait manifester 500 personnes, on était très fiers de nous. Ça durait dix minutes le temps que les flics arrivent et on se barrait. On avait des réseaux de motos qui nous prévenaient quand les flics arrivaient. On arrivait à faire des manifs en 10 minutes, impeccables, puis on se dispersait. Personne n'était piqué sauf les connards qui restaient pour discuter avec des gens pour avoir l'effet de la manif. C'est arrivé à certains, mais dans l'ensemble ça se passait très bien. On était très fiers de nous ${ }^{46}$.

45. Codaccioni V., «La construction d'une façade légaliste en contexte répressif : l'action anticoloniale communiste pendant le conflit algérien ", Sociétés Contemporaines, $\mathrm{n}^{\circ}$ 88, 2012, pp. $45-72$.

46. Entretien avec Alain Roux, Paris, 28 janvier 2008. 
Aux « héros » « victimes de la répression » capitaliste et bourgeoise de la guerre froide succèdent donc les «quelques connards » qui se font prendre par les policiers suite aux manifestations contre la guerre d'Algérie. C'est dire la dévalorisation, au sein du Parti, de la violence manifestante et, plus encore, de l'expérience vécue de la répression. Dès lors, dans ce contexte où la subir devient illégitime et où les arrestations sont perçues comme trop risquées car risquant d'entraîner l'isolement du Parti ou son interdiction, peu de communistes sont arrêtés dans le cadre de leurs activités militantes traditionnelles comme la distribution de tracts, le collage d'affiches ou les manifestations de rue ${ }^{47}$. Et même si certains d'entre eux sont arrêtés, ils sont pour la plupart libérés au bout de quelques heures ou de quelques jours sans susciter de grandes affaires comme cela avait été le cas pendant la guerre d'Indochine. Sur une cinquantaine d'enquêtés, seule Francette Lazard, née en 1937 et membre du PCF depuis 1952, raconte ainsi avoir été arrêtée dans le cadre de son militantisme quotidien, peu avant la manifestation du 13 mai 1958 :

«On collait des affiches pour la manif du 13 mai, on s'est fait emmener au poste. J'ai passé la nuit au poste, j'en ai un bon souvenir d'ailleurs [elle rit]. Le flic qui a relevé l'identité... il y avait un garçon qui était attaché de recherche au CNRS, et le flic a demandé : "Une police privée ?" [elle rit]. J'étais pliée de rire. Et puis... non, j'en ai gardé un souvenir. Le truc idiot c'est qu'on ne voulait pas demander d'aller aux toilettes, par dignité. C'était le commissariat du 6e. Mais je n'ai pas eu plus de répression. Ma seule expérience de répression, ça a été cette nuit en prison ${ }^{48}$.

Francette Lazard appartient à la génération de celles et ceux qui ont adhéré pendant la guerre froide et qui, s'ils ont été socialisés aux pratiques et aux discours de l'affrontement bipolaire, sont trop jeunes pour avoir connu personnellement et physiquement la répression dans les années cinquante. Dans un contexte de faible renouvellement du PCF et d'intensification des processus de vieillissement et de baisse de ses effectifs qui l'affectent - ils chutent de $60 \%$ par rapport à la période précédente ${ }^{49}$ - c'est donc ce type de membres, peu socialisés aux expériences répressives, qui forment la nouvelle

47. Ce qui ne veut pas dire qu'il n'y a pas la perpétuation d'un anticommunisme d'État, encore moins que l'on assiste à une disparition de la répression contre les membres du PCF. En témoignent la centaine de poursuites engagées contre ses journalistes ou ses journaux et, de manière paroxysmique, les morts de la manifestation Charonne du 8 février 1962 (Voir par exemple : Genevée F., «La répression: poursuites et saisies de L'Humanité», in Delporte C, Pennetier C., Sirinelli J-P et Wolikow S. (eds.), L'Humanité de Jaurès à nos jours, Paris, Nouveau monde Éditions, 2004, pp. 265-281; Dewerpe A., Charonne 8 février 1962 : Anthropologie historique d'un massacre d'État, Paris, Gallimard, 2006). Mais, à l'inverse de la période précédemment analysée, cette répression n'est plus anticipée, voulue et suscitée par le groupe dirigeant et les militants qui l'expérimentent personnellement et concrètement sont beaucoup moins nombreux.

48. Entretien avec Francette Lazard, Paris, le 11 janvier 2008.

49. Il atteint son plus faible score en 1960 et 1961 où il rassemble 300000 membres. 
base militante du parti et qui se mobilisent, de manière pacifique et légaliste, contre la guerre d'Algérie.

Néanmoins, si la stratégie d'abandon de la radicalité militante et l'arrêt de la ligne «ultra-gauche » qui avait prévalu jusqu'alors sont acceptés, intériorisés et réappropriés par la très grande majorité de ses membres, ils se heurtent à de multiples résistances au sein du PCF, notamment chez les plus jeunes militants qui risquent de partir combattre en Algérie dès 1955 au moment des rappels de soldats. Le cas de Jacques Bonnet est exemplaire à cet égard, lui qui relate en entretien sa " trouille énorme de partir » et sa déception face à la frilosité de l'engagement anticolonial communiste. Né en 1934 et membre du PCF depuis 1953, Jacques Bonnet termine ses études à l'ENSET Cachan au moment des rappels : "Je me disais : “demain, c'est ton tour” ». Il se souvient alors de ses actions pour empêcher le départ des rappelés :

«Les classes sont rappelées, il y a eu des mouvements importants là. $\mathrm{Tu} 50$ as eu des compagnies qui défilaient dans Paris, tu as eu des casernes qui se sont révoltées. Nous on avait fait des commandos de jeunes communistes, à la mode gauchiste, je dirais [il rit], et on était partis dans la région de Rouen à la caserne Richepanse, là-bas qui est à Rouen où les gars s'étaient révoltés, les rappelés, la caserne était encerclée par les CRS, nous on avait encerclé les CRS. Je me rappelle, à quelques-uns, on avait désarmé un CRS qu'on avait mis à poil, bon, rien du tout quoi. [...] On manifestait tous les jours. Et en même temps, on râlait contre le Parti qui ne faisait pas grand-chose ou contre la classe ouvrière qui ne bougeait pas des masses.

- C'étaient les jeunes qui...

- Mais voilà, on avait le sentiment que... Alors après on cherchait dans L'Huma, je me rappelle: “Mais nom de Dieu, hier, on s'est fait taper dessus sur le boulevard Bonne Nouvelle ou ici ou là”, rien ${ }^{51}$. »

La manifestation de Richepanse du 6 octobre 1955 est l'une des manifestations les plus violentes de l'année 1955, des pierres et des cocktails Molotov étant lancés contre les forces de l’ordre. Dix-huit «meneurs » sont déférés devant le tribunal militaire, accusés de " révolte militaire » et emprisonnés quelques semaines à Fresnes ${ }^{52}$. Ainsi, ce que cette manifestation révèle, c'est la radicalité des membres du PCF qui y participent, et en particulier celle des jeunes militants qui organisent des «commandos » « à la mode gauchiste » et affrontent les forces de l'ordre plus ou moins violemment. Or, et comme le dit Jacques Bonnet, le PCF passe sous silence ce type de manifestations, à la fois pour préserver l'image légaliste de son engagement contre la guerre d'Algérie,

50. Le tutoiement est courant dans les entretiens.

51. Entretien avec Jacques Bonnet déjà cité.

52. Jauffret J-C., Soldats en Algérie, 1954-1962 : expériences contrastées des hommes du contingent, Paris, Autrement, 2000, p. 33. 
pour amoindrir le coût d'une probable répression, mais aussi pour éviter une vague de radicalisation de ses membres les plus jeunes qui risquent de partir combattre. Contrairement à la période de guerre froide où offre et demande de répression se conjuguaient parfaitement au sein de l'organisation partisane, les plus jeunes membres du PCF connaissent lors du conflit algérien une « entrée en radicalité 53 » que le groupe dirigeant a du mal à contrôler. En l'occurrence, cette période du «temps des rappelés » est le moment de rupture pour de nombreux communistes qui préfèrent s'engager dans des organisations plus radicales, déserter ou s'insoumettre, des « désobéissants » à la ligne officielle qui sont les membres du Parti à subir la plus lourde répression pendant la guerre d'Algérie.

\section{Les "porteurs de valises " communistes: répression et désengagement militant}

À la différence de la guerre d'Indochine lors de laquelle le Parti jouait un rôle central dans les mobilisations anticoloniales, voire dominait à gauche l'interprétation et les mises en sens des conflits coloniaux, la guerre d'Algérie fait apparaître d'autres acteurs ou collectifs rapidement organisés en réseaux informels ou clandestins qui agissent sans le PCF voire contre lui. Leurs actions souvent illégales pour l'indépendance de l'Algérie les placent ainsi en situation d'avant-garde et modifient « le secteur 54 » du mouvement anticolonial, c'est-à-dire les systèmes d'alliances ou de conflits dans lesquels le Parti communiste est pris. Il en va ainsi du Parti communiste internationaliste (PCI) ${ }^{55}$ qui tente tout au long de la guerre de recruter ses militants critiques c'est la stratégie d'entrisme prônée par les trotskystes -, mais aussi du Front de libération nationale (FLN) et de ses réseaux d'aide en métropole au sein desquels s'engagent certains membres du PCF. Et s'ils appartiennent à toutes les générations militantes, les plus jeunes des « porteurs de valises » communistes font partie de la «génération des rappelés » directement confrontée à la guerre qui, pour militer de manière plus radicale contre le conflit algérien, se désengagent du PCF ou en transgressent les normes partisanes. Ils mettent alors en œuvre des modalités d'action condamnées par le groupe dirigeant comme l'hébergement de nationalistes algériens, le transport d'argent pour le FLN ou l'aide aux déserteurs, comme l'illustrent les trajectoires de Robert Bonnaud et de Louis Orhant.

Fils de communistes, nés dans les années trente, Robert Bonnaud et Louis Orhant font partie des militants qui ont adhéré au Parti communiste au plus fort de son isolement politique, respectivement en 1949 et 1951 . S’ils s'enga-

53. Romain Bertrand, "Plus près d'Allah”. L’itinéraire social et idéologique d'Imam Samudra ", in Collovald A., Gaïti B. (eds.), La démocratie aux extrêmes..., op. cit., p. 202.

54. Fillieule O., Péchu C., Lutter ensemble. Les théories de l'action collective, Paris, L'Harmattan, 1993, p. 177.

55. Sur l'action du Parti Communiste Internationaliste pendant la guerre d'Algérie, voir Pattieu S., Les camarades des frères. Trotskistes et libertaires dans la guerre d'Algérie, Paris, Éditions Syllepse, 2002. 
gent dans toutes les batailles communistes de la guerre froide, qui attachent les jeunes à l'organisation comme nous l'avons vu, l'année 1956 est celle de la rupture. Les événements critiques liés au début de la déstalinisation ${ }^{56}$, auxquels se superposent ceux inhérents à la radicalisation du conflit algérien comme la deuxième vague des rappels et le vote des pouvoirs spéciaux - «impardonnable 57 » pour Robert Bonnaud -, entrainent alors une "perte de foi dans le triomphe de la cause 58 » de l'anticolonialisme d'autant plus décisive pour ces militants qui risquent leur vie au combat. Rappelés en 1956 et refusant de combattre en Algérie, ils se retrouvent en effet en opposition frontale avec les cadres du PCF qui les incitent à partir selon le principe léniniste en vertu duquel « un militant communiste part à toutes les guerres, même réactionnaires ", son rôle étant de militer au sein de l'armée. Le « rappel » militaire opère alors comme une rupture biographique qui contraint ses membres soit au départ forcé sur le territoire algérien (Robert Bonnaud) soit à la désertion (Louis Orhant). Dans le premier cas, la confrontation directe et l'implication physique dans la guerre, outre qu'elle conforte l'anticolonialisme, engendre un fort ressentiment à l'égard de l'organisation partisane qui se traduit par une non reprise de carte l'année suivante. Dans le second cas, ce sont l'exil, la désertion, et le refus de combattre qui entraînent, après l'exit du territoire et du champ de bataille, celui du parti alors sanctionné par l'exclusion. Cette rupture biographique qu'est le rappel joue surtout comme un facteur de radicalisation politique et ce n'est pas un hasard si ces deux militants se spécialisent ensuite dans l'aide aux insoumis ou aux déserteurs, et qu'ils s'engagent dans des réseaux d'aide au FLN. Louis Orhant participe à la création et à l'activité d'un réseau d'aide aux déserteurs en Suisse, Robert Bonnaud, secrétaire de l'UGS ${ }^{59}$ dans les Bouches-du-Rhône, milite activement pour l'organisation frontiste et pour le soutien à ceux qui refusent de faire la guerre. Pour cette activité, ils sont largement réprimés : le premier est arrêté par la Direction de la surveillance du territoire (DST) le 21 janvier 1961 et condamné en août à deux ans d'emprisonnement pour désertion (sur dénonciation de son père), le second est arrêté en juin 1961, emprisonné, inculpé d'atteinte à la sûreté de l'État et libéré en juin 1962 après les accords d'Évian. Dans ce cas, la répression est donc intrinsèquement liée au processus de désengagement militant puisqu'elle n'intervient qu'une fois le militant libéré de son affiliation partisane, qu'il ait été exclu ou qu'il n'ait pas repris sa carte du parti. Néanmoins, il ne s'agit pas ici à proprement parler de désengagement militant mais plutôt de désaffiliation partisane ou de « sortie de rôle 60 » - celui du mili-

56. En particulier le rapport du XXe Congrès du Parti communiste de l'Union soviétique (PCUS) sur les «crimes de Staline » en février 1956 et l'écrasement de la Révolution hongroise par les troupes russes en novembre 1956.

57. Bonnaud R., Itinéraire, Paris, Les Éditions de Minuit, 1962, p. 16.

58. Leclercq C., " "Raisons de sortir". Les militants du Parti communiste français ", in Fillieule O., (ed.), Le désengagement militant, Paris, Belin, 2005, pp. 131-147.

59. Union de la Gauche Socialiste qui participe à la création du Parti socialiste unifié (PSU) en 1960.

60. Fillieule O., «Temps biographique, temps social et variabilité des rétributions », in Fillieule O., (ed.), Le désengagement militant, op. cit., p. 20. 
tant communiste discipliné -, le véritable désengagement n’intervenant que par une «mise hors jeu 61 » forcée comme l'emprisonnement, encore que le militantisme se poursuive à l'intérieur des prisons à l'exemple de Robert Bonnaud qui y dispense des cours. Le coût de ce militantisme radical est donc très lourd puisqu'il aboutit non seulement à une rupture avec le PCF mais surtout il se traduit par une répression policière ou militaire extrêmement dure qui se solde par une condamnation et un emprisonnement de longue durée.

\section{Les « refus communistes " et le débat sur la désobéissance militaire}

Le cas des réfractaires communistes, ceux qui refusent de porter les armes contre le peuple algérien en choisissant l'emprisonnement, est sensiblement différent puisque certains d'entre eux sont précisément choisis par la direction pour subir la répression à des fins anticoloniales. Après le premier « refus 62 » d'Alban Liechti ( $c f$. encadré) en septembre 1956 et les débats au sein du PCF entre les partisans de l'agitation au sein de l'armée et ceux favorables à la désobéissance militaire, le groupe dirigeant décide clandestinement l'année suivante de faire emprisonner des soldats communistes pour politiser la cause de la « Paix en Algérie ».

\section{Le premier « refus » et la transgression des normes du PCF : le cas d'Alban Liechti}

Né le 25 avril 1935, Alban Liechti grandit au sein d'une famille communiste mais contestataire, ce qui contribue à expliquer non seulement sa position hétérodoxe en 1956 mais aussi des petites protestations antérieures comme en 1953 où, à la mort de Staline, il n'en a « rien à foutre de faire le quart d'heure de grève pour Staline ». Prisonnier de guerre en 1940, son père télégraphiste aux PTT, est un " communiste contestataire » jusqu'à la seconde guerre mondiale. Sa mère, qu'il décrit comme "plus révolutionnaire que son père », adhère aussi au PCF en 1945, en même temps qu'elle milite au Secours populaire et à I'Union des Femmes dont elle devient rapidement responsable départementale pour la Seine-et-Oise. Mais il la décrit aussi comme une communiste " critique ", n'ayant jamais "encaissé " le culte de la personnalité et ayant refusé, en 1950 par exemple, alors qu'elle était secrétaire de cellule à Sèvres (où les Liechti habitent avec leurs neuf enfants), que sa cellule envoie un cadeau pour les cinquante ans de Maurice Thorez. Elle reçoit donc un blâme du Parti. Je crois comprendre par ailleurs, lors de l'un des deux entretiens qu'il m'accorde, que son père a aussi failli être exclu du Parti juste avant son départ pour l'Algérie. Alban Liechti, quant à lui, commence à vendre $L^{\prime}$ Humanité dès l'âge de 8 ans, adhère à 13 ans à I'Union des Jeunesses Républicaines et au PCF en 1955. Appartenant à la génération de la guerre froide, il fait partie des militants très actifs de cette période, comme en attestent ses multiples adhésions à des organisations satellites du PCF (France-URSS, Amis de Mitchourine, Mouvement de la Paix et Secours populaire). Il est d'ailleurs, comme nombre de ses camarades, matraqué, hospitalisé et inculpé à la suite de la manifestation

61. Ibid., p. 19.

62. Quemeneur T., Une guerre sans non? Insoumissions, refus d'obéissance et désertion de soldats français pendant la guerre d'Algérie (1954-1962), thèse pour le doctorat d'histoire, Université Paris VIII, 2008. 
Ridgway, un " baptême du feu 63 " sur lequel il revient lors de nos deux entretiens, témoignant là encore de la manière dont la répression est vécue comme un "fait d'armes » au service de la cause du communisme dont on peut parler avec fierté. Et après son départ en Algérie en septembre 1956, Alban Liechti est condamné une première fois le 19 novembre 1956 à deux ans de prison pour " désobéissance militaire ». Puis, face à son nouveau refus, il est condamné à nouveau le 26 mai 1959 à deux ans de prison. Au total, il connaît donc quatre ans d'emprisonnement pour avoir refusé de porter les armes contre le peuple algérien.

Parmi les trente-trois «soldats du refus » se trouve d'ailleurs «l'héritier» Serge Magnien 64. Devenu dirigeant de l'UEC en 1956 et déjà fiché par l'armée ${ }^{65}$ dans un contexte de radicalisation de la « chasse au rouge » au sein de l'institution militaire, il est condamné en 1958, comme les autres réfractaires, à deux ans d'emprisonnement pour « refus d'obéissance». Or, en mai 1959, Maurice Thorez décide de renoncer à cette modalité d'action, témoignant du renforcement des positions légalistes au sein du PCF, en partie induit par les victoires électorales obtenues par le Parti aux élections législatives de mars 1959. Mais l'arrêt de cette radicalisation clandestine et souterraine doit aussi être mise en lien avec la création, justement en mai 1959, d'une organisation à laquelle les jeunes membres du PCF ont interdiction d'adhérer : Jeune Résistance (JR).

Animée par une dizaine de déserteurs communistes oppositionnels dont Louis Orhant ou par des militants de la nouvelle gauche, JR a pour but d'encourager collectivement la désobéissance militaire, comme l'illustre la publication de l'ouvrage Le Déserteur de Maurienne (Jean-Louis Hurst, un instituteur communiste et l'un des fondateur de Jeune Résistance) qui illustre le cheminement d'un soldat, fidèle au PCF, mais qui s'en détache peu à peu pour déserter. Car Jeune Résistance critique dès sa création le PCF, et en particulier la simple désobéissance militaire de ses membres à laquelle elle oppose tout au long du conflit ses propres méthodes, plus radicales, et notamment ce que Maurice Maschino appelle « le refus hors la loi 66 » c'est-à-dire la désertion.

«Actuellement, vu le désintéressement de l'opinion française et l'apathie de la gauche, écrit-il dans Le refus, il est tout à fait inefficace de moisir en taule ; bien sûr si chaque refus devenait une affaire Henri Martin [...] ça vaudrait probablement la peine de se constituer prisonnier, ce n'est pas le cas ${ }^{67}$.

63. Selon son expression (Entretiens avec Alban Liechti, le 12 décembre 2006 et le 27 février 2007, Trappes).

64. En entretien, il précise que son geste n’a pas été dicté par les dirigeants du Parti.

65. Arch. SHD, 10T515. Note de renseignements du 5 mars 1958.

66. Maschino M., L'engagement, Paris, Maspero, 1961, p. 23.

67. Maschino M., Le refus, Paris, François Maspero, 1960, p. 9. 
Ici, c'est le rapport du PCF à la répression et plus précisément encore le choix de l'emprisonnement politique qui est dénoncé pour son inefficacité. Cette mise en cause des « refus », qui délégitime et dévalorise la modalité d'action la plus radicale mise en œuvre clandestinement par les communistes, témoigne de la manière dont le PCF est dépassé sur sa gauche par d'autres types d'organisations qui le contraignent, dans une volonté de démarcation et par crainte de la répression, à une stratégie de repli légaliste. Car même si ses effectifs ne dépassent pas huit cent membres ${ }^{68}$, JR noue des relations avec d'autres organisations favorables à l'indépendance de l'Algérie ${ }^{69}$, permet des passages entre réseaux d'aide aux déserteurs et réseaux d'aide au FLN, et participe d'une plus grande diffusion du thème de l'insoumission à la politique coloniale de la France. L'organisation représente aussi une force politique nouvelle qui rallie de plus en plus de jeunes, comme Alain Krivine qui, né en 1941 et membre de l'UEC en 1956 dont il devient l'un des dirigeants l'année suivante, adhère parallèlement et clandestinement à JR par l'intermédiaire de ses deux frères, tous deux trotskistes et passés à l'aide au FLN. En son sein, il mène alors trois types d'actions clandestines - le blocage des trains de soldats en partance pour l'Algérie, l'aide au transport d'argent pour le FLN et l'aide aux évasions de militants frontistes emprisonnés en métropole :

«Je m'occupais de l'arrêt des trains, il y avait des déserteurs qui partaient en Suisse donc je m'en occupais. On avait des contacts cheminots donc on bloquait les trains. Ça faisait du bruit, j'étais fiérot, j’entendais à la radio Jeune Résistance vient encore de bloquer des trains [...]. J'étais jeune, ça m'excitait, on faisait passer des bagnoles pleines de fric pour le FLN. On était postés tous les 100 mètres et s'il y avait les flics, on devait faire son nœud de chaussure. C'étaient des conneries, mais ça marque, je ne fais pas ça tous les jours (...). J'ai aidé à la Roquette, j'étais une petite main. C'était agréable. J'étais dans les bras d'une nana, et on devait se bisouter sur les talus pour repérer les rondes de flics, c'est sympa ça. Donc j'étais volontaire. Et moi ça me rappelait... J'ai toujours été là-dedans, la Résistance, les machins, les trucs, et j'ai toujours été un peu activiste, je trouvais ça bien ${ }^{70}$. »

Ce qui se donne à voir dans ce témoignage, ce sont les rétributions militantes à l'œuvre dans le cadre de l'action clandestine pour l'indépendance de l'Algérie : l'impression de continuer les grandes luttes historiques - et la référence à la Résistance n'est pas anodine ici puisqu'elle est centrale dans les discours anticolonialistes de la période -, la possibilité de faire de nouvelles ren-

68. Liauzu C., «Ceux qui ont fait la guerre à la guerre », in Stora B., Harbi M. (eds.), La guerre d'Algérie : 1954-2004, Paris, Robert Laffont, 2004, p. 162.

69. Comme les réseaux Jeanson et Curiel, l’Union Générale des Etudiants Musulmans Algériens, branche étudiante du FLN, etc.

70. Entretien avec Alain Krivine, Montreuil, le 4 avril 2012. 
contres amicales ou amoureuses, la fierté de voir ses actions relayées dans les médias, l'excitation procurée par les pratiques militantes «à risque » ou, de manière plus ludique, les sortes de jeux de « cache-cache » avec les forces de l'ordre. Car ce qui compte pour les jeunes qui désobéissent au groupe dirigeant du PCF pendant la guerre d'Algérie, c'est la quotidienneté et la diversité des actions interdites (par la loi et par « le Parti »), l'une des seules manières de se représenter encore comme des « activistes ». Ce n'est d'ailleurs pas un hasard si, au moment de l'intensification des affrontements avec l'Organisation de l'armée secrète (OAS), Alain Krivine s'engage au Front Universitaire Antifasciste (FUA), regroupant des dissidents de l'Union nationale des étudiants de France (UNEF), du Parti socialiste unifié (PSU) ou du PCF qui appliquent des méthodes d'action directes (des commandos notamment) pour éradiquer l'extrême droite du Quartier Latin ${ }^{71}$, et qui sont là encore désavoués et dénoncés par la direction communiste comme "gauchistes ». Alain Krivine fait donc partie des militants (et des dirigeants dans son cas) de l'UEC qui soutiennent la désertion et l'insoumission, qui passent au soutien direct à l'organisation frontiste et qui choisissent d'autres types d'organisations militantes pour réagir au « manque de radicalité, d'anticolonialisme et d'internationalisme » $\mathrm{du}$ PCF 72.

Dans les périodes de radicalisation, qui sont aussi celles de l'isolement politique, la répression touche l'ensemble des membres du PCF (dirigeants, intellectuels, cadres ou jeunes militants) et joue un rôle central dans le fonctionnement de l'institution communiste. Non seulement elle renforce la solidarité partisane contre les agents répressifs et autour de la cause des « victimes de la répression » capitaliste et bourgeoise, mais elle permet au groupe dirigeant de socialiser les plus jeunes d'entre eux à la discipline de parti et au rôle du communiste des périodes de repli. Elle permet ainsi à l'organisation de disposer de militants plus « sûrs » car ayant intériorisés l'orthodoxie et l'orthopraxie communistes, tandis que pour ces derniers, la répression est progressivement intégrée comme un événement partie intégrante de leur vie militante vecteur de gratifications. A contrario, dans les séquences légalistes qu'illustre la guerre d'Algérie, les militants les plus réprimés sont ceux qui transgressent les normes partisanes ou qui se désengagent du PCF, choisissant d'autres causes (l'indépendance de l'Algérie, le soutien aux déserteurs et aux insoumis) et d'autres comportements militants (l'action clandestine ou radicale) que ceux proposés et imposés par leur parti. Certains jeunes militants en particulier, et notamment ceux formés au sein de l'UEC, optent alors pour d'autres types d'engagement, plus conformes à leurs attentes et dans lesquels les rétributions sont plus immédiates. Car ce n'est pas l'absence de répression anticommuniste à proprement parler qui crée les conditions du désengagement de

71. Fischer D., Les étudiants en France (1945-1968). Contribution à une histoire socio-culturelle et politique $d u$ milieu étudiant, Thèse pour le doctorat d'histoire, Université Paris XNanterre, 1998, p. 518.

72. Entretien avec Alain Krivine déjà cité. 
nombreux militants du PCF pendant le conflit algérien, mais l'abandon des pratiques et des discours du militant « révolutionnaire » cycliquement réprimé : la défense de causes marginales, l'activisme en marge ou en transgression de la loi, la conflictualité avec les agents de l'État ou encore la croyance en la possibilité d'instaurer la dictature du prolétariat. On assiste dès lors à un dérèglement du système de rétributions de ses franges les plus radicalisées qui, dès le milieu des années soixante, intègrent des organisations concurrentes du PCF, notamment dans ses prétentions à faire la « révolution ${ }^{73}$ », comme la Jeunesse Communiste Révolutionnaire créée par Alain Krivine, l'Union des Jeunesses Communistes marxistes-léninistes ou après mai 68, la Gauche Prolétarienne ${ }^{74}$. Ainsi, ce dont témoigne plus généralement le hiatus entre le groupe dirigeant du PCF et ses jeunes les plus radicaux pendant la guerre d'Algérie, c'est la manière dont le légalisme du PCF participe à la dépréciation de l'activisme communiste ou, pour le dire comme Boris Gobille à propos de mai 1968, à une « démonétisation brusque et massive du PCF à la bourse des valeurs de la radicalité 75 ». Et si cette stratégie légaliste n'est pas inédite dans l'histoire du Parti communiste - on la retrouve notamment à la Libération lorsque le groupe dirigeant tente de capitaliser les diverses ressources obtenues sous l'Occupation -, elle a la particularité de provoquer des oppositions, des dissidences et des ruptures ${ }^{76}$, et d'exclure durablement le PCF des réseaux de l'avant-garde militante. Les événements de mai-juin 1968 s'inscrivent d'ailleurs dans la stricte continuité de la gestion thorézienne de la guerre d'Algérie puisque le groupe dirigeant opte, après de nombreuses hésitations tactiques, pour la voie législative et la stratégie d'accession au pouvoir par les urnes ${ }^{77}$. Néanmoins, cette stratégie de disqualification des «gauchistes » et de leurs pratiques, alliée à l'accentuation sur le " caractère pacifique du passage au socialisme 78 », se révèle payante pour l'organisation : ses effectifs recommencent à crô̂tre dès le début des années soixante et le PCF attire un électorat et une base militante plus proches du profil de la population française. Ce sont dès lors des effectifs plus jeunes, plus féminins et moins ouvriers qui viennent grossir les rangs du Parti, tandis que ce dernier recueille 22,5 \% des suffrages exprimés aux élections législatives de 1967, résultats confirmés par les présidentielles de 1969 où Jacques Duclos obtient $21,5 \%$ des voix au premier tour. Ainsi, non seulement le décrochage

73. Boulland P., Ethuin N., Mischi J., «Les disqualifications des gauchistes au sein du PCF. Enjeux sociologiques et stratégiques », Savoir/agir, n 6, décembre 2008, p. 31.

74. Matonti F., Pudal B., "L’UEC ou l'autonomie confisquée », in Damamme D., Gobille B., Matonti M., Pudal B., (eds.), Mai-juin 68, Paris, Les Éditions de l’Atelier, 2008, p. 141.

75. Gobille B., «Les mobilisations de l'avant-garde littéraire en mai 1968. Capital politique, capital littéraire et conjoncture de crise ", Actes de la recherche en sciences sociales, $2005, \mathrm{n}^{\circ} 258$, p. 35 .

76. En 1945 en effet, le légalisme imposé par Maurice Thorez suscite des résistances à tous les échelons du Parti, mais elles ne se traduisent que très rarement par le départ de l'organisation (Codaccioni V., Punir les opposants..., op. cit., pp. 113-120).

77. Gobille B., «L’événement mai 1968. Pour une sociohistoire du temps court », Annales. Histoires, Sciences sociales, $2008, \mathrm{n}^{\circ} 2$, p. 347.

78. Pudal P., Un monde défait..., op. cit., p. 81. 
anticipé de cette jeunesse radicalisée est comblé par une nouvelle génération militante peu tentée par la gauche du PCF, mais surtout le « gauchisme ne fissure pas 79 » et déstabilise peu, pour l'heure, l'institution communiste.

79. Martelli R., Prendre sa carte. 1920-2009. Données nouvelles sur les effectifs du PCF, Seine Saint-Denis, Fondation Gabriel Péri, 2010, p. 36. 